ISSN 2073-4360

www.mdpi.com/journal/polymers

Article

\title{
Microspheres Containing Cibacron Blue F3G-A and Incorporated Iron Oxide Nanoparticles as Biomarker Harvesting Platforms
}

\section{Alexis Patanarut ${ }^{1}$, Elissa H. Williams ${ }^{1}$, Emanuel Petricoin ${ }^{2}$, Lance A. Liotta ${ }^{2}$ and Barney Bishop ${ }^{1, *}$}

1 Department of Chemistry, George Mason University, 4400 University Drive, MSN 3E2, Fairfax, VA 22030, USA; E-Mails: spatanar@gmu.edu (A.P.); ewilliah@gmu.edu (E.H.W.)

2 Center for Applied Proteomics and Molecular Medicine, George Mason University, 10900 University Blvd, MSN 1A9, Manassas, VA 20110, USA; E-Mails: epetrico@gmu.edu (E.P.); 1liotta@gmu.edu (L.A.L.)

* Author to whom correspondence should be addressed; E-Mail: bbishop1@gmu.edu; Tel.: +1-703-993-8302.

Received: 8 June 2011; in revised form: 11 July 2011 / Accepted: 27 July 2011 /

Published: 28 July 2011

\begin{abstract}
In this work, magnetic functionality was introduced to cross-linked acrylamide-based particles via the in situ coprecipitation of iron oxide nanoparticles within the hydrogel particle interior. Cibacron Blue F3G-A was then incorporated onto the magnetic hydrogel scaffold to facilitate the harvest of targeted protein species. The dye-loaded magnetic particles were physically characterized, and their protein sequestration performance was investigated. The results of these studies indicated that dye-loaded magnetic particles sequestered a greater amount of lower molecular weight proteins from the test solution than was achieved using reference particles, dye-loaded cross-linked N-isopropylacrylamide-based core-shell particles. This difference in protein harvesting ability may reflect the higher degree of dye-loading in the magnetic particles relative to the dye-loaded core-shell particles.
\end{abstract}

Keywords: hydrogels; Cibacron Blue F3G-A; iron oxide nanoparticles; affinity dyes 


\section{Introduction}

Low-abundance, low-molecular weight peptides, proteins and other biomolecules, collectively known as biomarkers, can provide important information about the physiological state of an organism. By studying biomarkers, diseases such as cancer can be diagnosed in its early stages, thereby improving the overall prognosis for the patient. However, the presence of high-molecular weight, high-abundance species such as immunoglobulins and serum albumin can hinder the separation and detection of biomarkers. Therefore, biomarker sequestration platforms must have the ability to harvest dilute amounts of target analyte out of complex mixtures and concentrate the analyte to detectable levels. Cross-linked N-isopropylacrylamide (pNIPAm)-based hydrogel particles with incorporated affinity baits acrylic acid (AAc) and Cibacron Blue F3G-A have shown an ability to rapidly, in one step, sequester, concentrate and protect from proteolytic degradation low molecular weight proteins and peptides from serum, thereby allowing the analysis of low-abundance labile biomarkers $[1,2]$. The cross-linked polymer chains that make up the hydrogel particle provide a framework with a significant degree of flexibility and porosity, and the highly solvated polymer network allows the small molecules access into the interior of the particle. The presence of the affinity baits incorporated within the polymer chain facilitates the capture of targeted analytes and prevents their diffusion out of the particle (Figure 1) [1].

Figure 1. Sequestered low molecular weight species can diffuse out of underivatized hydrogel particles (A); Incorporation of affinity bait within the particle matrix enables the particle to effectively retain the harvested low molecular weight species and prevent them from escaping the particle matrix $(\mathbf{B})$.
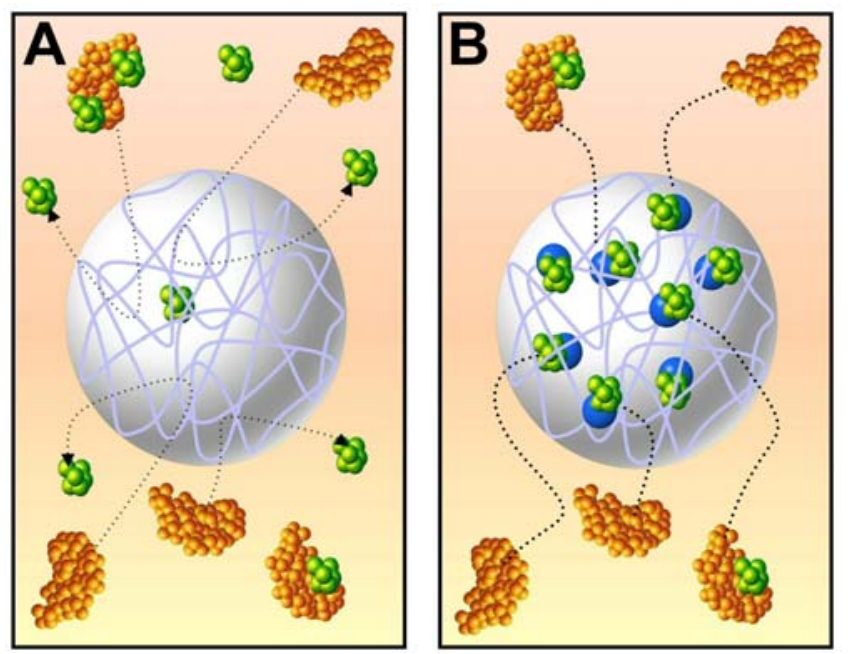

Hydrogel particles currently in use for biomarker harvesting are collected from the sample and wash solutions by means of centrifugation, which complicates their use in automated processes and high-throughput applications. Incorporation of magnetic elements within the affinity-baited hydrogel particle architecture will further enhance their utility in adsorption/harvesting applications due to the ease and rapidity with which magnetic particles can be separated from complex mixtures by utilizing an external magnetic field. Magnetic decantation is less time-consuming than traditional separation techniques such as centrifugation and filtration. In addition, target analytes sequestered and separated 
by magnetic particles are subjected to very low mechanical stress in comparison to centrifugation or filtration methods [3].

Several methods have been reported for the preparation of magnetic particles, however not all of these approaches yield particle architectures that are well suited for harvesting applications. One approach involves assembling inorganic nanoparticle composites on the surface of an organic polymer bead via covalent attachment [4], or Pickering emulsion [5] to yield hybrid materials with an organic hydrogel core encased within an inorganic iron oxide shell. However, the iron oxide shell may complicate the sequestration of low molecular weight species by hindering the target molecules from entering the porous hydrogel particle. Another commonly used approach involves polymerizing a monomer mixture in the presence of surfactant-coated inorganic magnetic nanoparticles via emulsion and microemulsion polymerization. However, because the reaction is performed in the presence of surfactant-coated iron oxide nanoparticles, incomplete or nonuniform encapsulation of the iron oxide nanoparticles is a possibility. Furthermore, the reaction parameters have to be optimized to control particle size and achieve complete and uniform incorporation of the inorganic nanoparticles within the resultant polymer bead. In the third approach, developed by Ugelstad et al., the iron oxide nanoparticles were coprecipitated under alkaline conditions inside the pores of preformed monodisperse polymer particles [6]. This approach affords porous magnetic hydrogel particles with uniform incorporation of iron oxide nanoparticles within the particle network.

We report here the design, synthesis and characterization of hydrogel particles impregnated with magnetic iron oxide nanoparticles and baited with Cibacron Blue F3G-A. Cross-linked acrylamide-based hydrogels are commonly used as templates for the in situ generation of magnetic nanoparticles, since polymer adsorption studies have established that acrylamide-based polymers interact with a range of metal cations in solution [7]. The porous nature of the hydrogel particles allows the aqueous iron salts to diffuse freely throughout the polymer matrix, and amide $\left(\mathrm{CONH}_{2}\right)$ groups present in the polymer framework can bind the iron (II) and iron (III) cations via electrostatic interactions [8]. Magnetic nanoparticles are then formed within the hydrogel matrix via coprecipitation of the iron salts, and these iron oxide nanoparticles are bound tightly to the pAm hydrogel via hydrogen bonding interactions between the polymer and the iron oxide nanoparticles $[9,10]$.

In order to affix the affinity bait, Cibacron Blue F3G-A, to the hydrogel scaffold, it was necessary to introduce suitable functionalizable groups into the polymer framework. This was achieved by subjecting the magnetized acrylamide particles to controlled Hofmann degradation, which effectively converted a fraction of the acrylamide carboxamide groups to primary amines. These amines were in turn derivatized with Cibacron Blue F3G-A using conditions that were previously developed for the synthesis of NIPAm based dye-loaded particles [2]. The swelling and physical properties of the resulting baited magnetic particles were characterized, and their ability to effectively harvest and concentrate low abundance peptides and proteins while excluding larger higher molecular weight proteins was evaluated. 


\section{Experimental Section}

\subsection{Materials}

Acrylamide (Am), N-N'-methylenebis(acrylamide) (BIS), azobisisobutyronitrile (AIBN), dextrain, iron (II) chloride tetrahydrate and iron (III) chloride hexahydrate were purchased from Sigma-Aldrich, Corp. Cibacron Blue F3G-A was purchased from Polysciences, Inc. All reagents were used as received. 18\% Tris-Glycine gels and Tris-Glycine SDS running buffer were purchased from Invitrogen Corporation. Water for all reactions, solution preparation, and polymer washing was distilled/purified using a Millipore Milli-Q water purification system to a resistance of $18 \mathrm{M} \Omega$ and passed through a $0.2 \mu \mathrm{m}$ nylon filter.

\subsection{Synthesis of Cross-Linked pAm Particles}

Hydrogel particles based on pAm were prepared via precipitation polymerization in ethanol [11]. Am (36.0 mmol, $2.56 \mathrm{~g}$ ) and BIS (14.0 mmol, $2.16 \mathrm{~g}$ ) were dissolved in $100 \mathrm{~mL}$ of ethanol, and the solution was transferred to the reaction flask. The solution was purged with $\mathrm{N}_{2}$ at room temperature with vigorous stirring for $30 \mathrm{~min}$ to remove dissolved oxygen. After degassing with $\mathrm{N}_{2}$, the reaction was heated to $70{ }^{\circ} \mathrm{C}$. Once the reaction mixture had attained a stable temperature of $70{ }^{\circ} \mathrm{C}$, AIBN $(0.353 \mathrm{mmol}, 0.0840 \mathrm{~g})$ in $1.0 \mathrm{~mL}$ of ethanol was added to initiate the polymerization. The reaction was maintained at a constant temperature of $70{ }^{\circ} \mathrm{C}$ with vigorous stirring under $\mathrm{N}_{2}$ for $3 \mathrm{~h}$. After this time, the reaction was allowed to cool to room temperature with stirring under $\mathrm{N}_{2}$. The particles were then harvested and washed with ethanol and water via centrifugation (Eppendorf 5415R centrifuge). In this process, $1.0 \mathrm{~mL}$ aliquots of particles were centrifuged for $20 \mathrm{~min}$ at $23{ }^{\circ} \mathrm{C}$ and 16,100 relative centrifugal force (rcf), with the supernatant subsequently discarded. Each of the particle pellets were

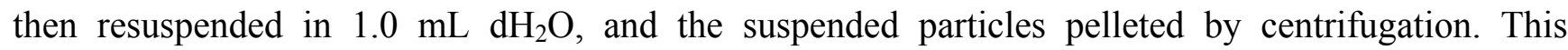
centrifugation/redispersion process was repeated for a total of five times. Following the fifth centrifugation step, the harvested particles were allowed to dry overnight under vacuum.

\subsection{Synthesis of Magnetic Hydrogel Particles}

The preparation of magnetic hydrogel microspheres based on a copolymer of Am and methacrylic acid (MAA) has been previously described [12]. The protocol described here addresses the in situ coprecipitation of iron oxide nanoparticles within the polymeric network of pAm hydrogel particles.

Dried pAm hydrogel particles $(0.200 \mathrm{~g})$ were rehydrated in an aqueous solution ( $4.0 \mathrm{~mL}$ volume) containing $0.400 \mathrm{~g}$ dextran and a 1:2 molar ratio mixture of iron (II) chloride tetrahydrate $(1.29 \mathrm{mmol}$, $0.256 \mathrm{~g})$ and iron (III) chloride hexahydrate $(2.23 \mathrm{mmol}, 0.604 \mathrm{~g})$. The hydrogels were allowed to equilibrate for $3 \mathrm{~h}$ with stirring and gentle heating at $60{ }^{\circ} \mathrm{C}$. After $3 \mathrm{~h}$, the reaction was allowed to cool to room temperature, and $4.0 \mathrm{~mL}$ of $7 \%$ ammonium hydroxide was then added quickly to the reaction vessel to coprecipitate the iron salts. The magnetic hydrogels were then harvested and washed with water via centrifugation. In this process, $1.0 \mathrm{~mL}$ aliquots of the particles were centrifuged for $20 \mathrm{~min}$ at 6,000 (rcf) to separate the hydrogels from the bulk aqueous medium containing the noncomposite iron oxide nanoparticles. The supernatant for each sample was subsequently removed and discarded. 
Each aliquot of pelleted particles was then resuspended in $1.0 \mathrm{~mL} \mathrm{dH}_{2} \mathrm{O}$, and the suspended particles were pelleted by centrifugation. This centrifugation/redispersion process was repeated until the supernatant was clear. Following the final centrifugation step, each aliquot of the particles was resuspended in $1.0 \mathrm{~mL}$ of $\mathrm{dH}_{2} \mathrm{O}$ and stored as a suspension. These magnetic hydrogel particles were named 100MHP. This procedure was also employed to prepare magnetic hydrogel particles using 50\% and $75 \%$ of the iron salts used in the described protocol. These were named 50MHP and 75MHP, respectively (Table 1).

Table 1. Amount of iron salts used to prepare magnetic hydrogels with different iron loadings.

\begin{tabular}{cccc}
\hline Sample No. & Hydrogel type & Fe (II) (mg) & Fe(III) (mg) \\
\hline 1 & $50 \mathrm{MHP}$ & 0.128 & 0.301 \\
2 & $75 \mathrm{MHP}$ & 0.192 & 0.444 \\
3 & $100 \mathrm{MHP}$ & 0.256 & 0.604 \\
\hline
\end{tabular}

The iron content of the magnetic hydrogel particles was determined by dividing the iron oxide weight by the total dry weight of the magnetic hydrogel particles of a given sample. The total mass of iron oxide per gram of dried magnetic hydrogel was calculated by the difference between the weight of the dried magnetic hydrogel and the weight of the dried pAm hydrogel used in the reaction.

\subsection{Hofmann Degradation of Magnetic Hydrogel Particles}

The protocol described here addresses the introduction of amino groups into the pAm-based submicron hydrogel microspheres via Hofmann degradation using similar reaction parameters that have been previously described [13]. A $4.0 \mathrm{~mL}$ aliquot of magnetic pAm hydrogel particles suspended

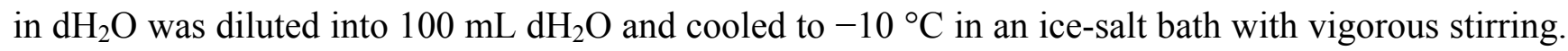
The reaction was performed under $\mathrm{N}_{2}$ to minimize the occurrence of side reactions. Ice-cooled $5 \%$ sodium hypochlorite ( $8.0 \mathrm{~mL}$ volume) and $2.4 \mathrm{wt} \%$ sodium hydroxide (12 mL volume) were slowly added to the reaction and stirred. The reaction was kept at $-10{ }^{\circ} \mathrm{C}$ with vigorous stirring for $1.5 \mathrm{~h}$. Ice-cooled $2.4 \mathrm{wt} \%$ sodium hydroxide was then slowly added to the particle suspension. The reaction was kept at $-10{ }^{\circ} \mathrm{C}$ under vigorous stirring for an additional $30 \mathrm{~min}$. After $30 \mathrm{~min}$, the reaction was transferred to an ice- $\mathrm{H}_{2} \mathrm{O}$ bath, in which the reaction warmed to $0{ }^{\circ} \mathrm{C}$. The reaction was kept at $0{ }^{\circ} \mathrm{C}$ for $4 \mathrm{~h}$, after which the aminated magnetic hydrogels were harvested via magnetic decantation and washed several times with $\mathrm{dH}_{2} \mathrm{O}$. After the final wash, the aminated magnetic hydrogels were stored in $\mathrm{dH}_{2} \mathrm{O}$ as a suspension.

\subsection{Immobilization of Cibacron Blue F3G-A}

The incorporation of Cibacron Blue F3G-A in pNIPAm-co-AA core particles has been previously described [14]. The protocol described here addresses the incorporation of Cibacron Blue F3G-A in the amine functionalized magnetic hydrogel particles. Cibacron Blue F3G-A (0.843 mmol, $0.708 \mathrm{~g})$ was dissolved in $8 \mathrm{~mL}$ of $0.1 \mathrm{M}$ aqueous sodium carbonate. A $4.0 \mathrm{~mL}$ aliquot of amine functionalized magnetic hydrogel particles suspended in $\mathrm{dH}_{2} \mathrm{O}$ was pelleted by centrifugation. Following centrifugation, the pelleted particles were resuspended in $4.0 \mathrm{~mL}$ of $0.1 \mathrm{M}$ aqueous sodium carbonate 
and purged with $\mathrm{N}_{2}$ for 15 min at room temperature with stirring in the reaction flask. After degassing with $\mathrm{N}_{2}$, the dye solution was added, and the combined reaction mixture was allowed to continue stirring at room temperature under nitrogen for $48 \mathrm{~h}$. After this time, $1 \mathrm{~mL}$ aliquots of blue particles were centrifuged for $20 \mathrm{~min}$ at $23{ }^{\circ} \mathrm{C}$ and 16,100 rcf. The supernatants were decanted and each tube of particles was redispersed in $1.0 \mathrm{~mL}$ of $\mathrm{dH}_{2} \mathrm{O}$. This centrifugation/redispersion process was repeated until the supernatant was clear. Following the final centrifugation step, the particles were resuspended in $1.0 \mathrm{~mL}$ of $\mathrm{dH}_{2} \mathrm{O}$ and stored as a suspension.

\subsection{Swelling Studies}

The swelling behavior of the pAm core, magnetic core, amine functionalized magnetic core and dye-loaded magnetic core particles were investigated as a function of $\mathrm{pH}$. All swelling studies were conducted at room temperature. The particles were first lyophilized to remove the water from the particle matrix, then weighed (recorded as dry weight). The samples were then immersed in $1.0 \mathrm{~mL}$ of swelling solvent $\left(\mathrm{dH}_{2} \mathrm{O}\right)$ and gently agitated on a nutator for $24 \mathrm{~h}$. After $24 \mathrm{~h}$, the samples were centrifuged for $20 \mathrm{~min}$ at $16,100 \mathrm{rcf}$ to separate the particles from the bulk aqueous medium. The supernatant for each sample was removed and discarded. The resultant pellets were blotted quickly with a soft tissue to remove any remaining surface droplets and weighed (recorded as wet weight). The degree of hydration of the particles, in terms of percentage of total mass of hydrated particles attributable to water, was calculated from the average masses of the particles dry $\left(\mathrm{W}_{\mathrm{dry}}\right)$ and hydrated $\left(\mathrm{W}_{\text {wet }}\right)$ using Equation 1 below [15].

$$
\text { [Swelling ratio } \left.=\mathrm{W}_{\text {wet }} / \mathrm{W}_{\text {dry }}\right]
$$

\subsection{Scanning Electron Microscopy (SEM)}

To determine the morphologies of the magnetic hydrogel microspheres, scanning electron microscopy (Hitachi S-4700 High Resolution Field-Emission, Japan) was performed under ultra-high resolution and magnetic mode, 40 microamperes, and a voltage of $15 \mathrm{keV}$. The samples were prepared by placing an aliquot of particle suspension in $\mathrm{dH}_{2} \mathrm{O}$ on a silicon wafer and allowing it to dry at room temperature. One sample from each batch was randomly selected for scanning.

\subsection{Protein Sieving and Harvesting Studies}

The protein binding and sieving properties of the particles were evaluated by incubating the particles in a solution containing a combination of proteins with molecular weights ranging from 6,500 to $66,000 \mathrm{kDa}$. The protein solution consisted of $0.5 \mathrm{mg} / \mathrm{mL}$ of each of the following proteins: aprotinin (MW 6,500 Da, pI 10.5, Sigma Aldrich), lysozyme (MW 14,400 Da, pI 11.1, Sigma Aldrich), trypsin inhibitor (MW 21,500 Da, pI 4.6, Invitrogen), carbonic anhydrase (MW 31,000 Da, pI 6.0, Sigma Aldrich), ovalbumin (MW 45,000 Da, pI 4.6, Sigma Aldrich), and BSA (MW 66,000 Da,

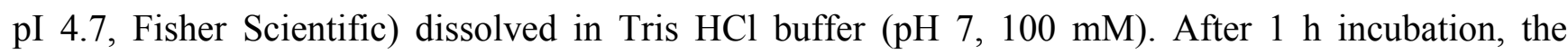
samples were centrifuged at 16,100 ref for $7 \mathrm{~min}$ to separate the particles from the aqueous medium. The pelleted particles were washed three times with $1.0 \mathrm{~mL}$ aliquots of water, and the captured 
proteins were electroeluted onto an SDS polyacrylamide gel (18\% Tris-glycine, Invitrogen) at $200 \mathrm{~V}$ for $1 \mathrm{~h}$. The gels were silver stained.

Image analysis of each gel was performed by capturing the image of each gel with a PC scanner (HP ScanJet 5400c) and saving it as graphic files in JPEG format. Densitometric analysis was performed using gel analysis software (ImageJ for Windows).

\section{Results and Discussion}

\subsection{Particle Synthesis and Physical Characterization}

\subsubsection{Preparation of Magnetic Hydrogels}

Hydrogel microspheres synthesized by precipitation polymerization typically have uniform size distributions and are free of contaminants. In precipitation polymerization systems, the final polymer is insoluble in the monomer-solvent mixture and is precipitated out of solution as it is formed. Therefore, ethanol is commonly used as the solvent since acrylamide is soluble in ethanol $(86.2 \mathrm{~g} / 100 \mathrm{~g}$ ethanol, $\left.30{ }^{\circ} \mathrm{C}[16]\right)$ but the resultant polymer is insoluble [17]. To this effect, homogeneous pAm microspheres were synthesized using a surfactant-free precipitation polymerization system with ethanol as the reaction medium, BIS as the cross-linker, and AIBN as the initiator [12].

Magnetic hydrogels were obtained by the in situ coprecipitation of iron (II) chloride tetrahydrate and iron (III) chloride hexahydrate to form the resultant magnetite iron oxide nanoparticles within the hydrogel. The porous nature of the pAm hydrogel particles allow for the iron nanoparticles to deposit within the polymeric matrix, and the amide $\left(\mathrm{CONH}_{2}\right)$ groups in the polymer chains bind, via electrostatic interactions, the iron (II) and iron (III) chloride species in the aqueous solution of iron (II) chloride tetrahydrate and iron (III) hexahydrate [8]. Formation of the magnetic nanoparticles within the hydrogel particles is easily monitored via the color change of the swollen hydrogel during the in situ coprecipitation process. The iron ions were loaded into the hydrogel using the method described by Kawaguchi et al., in which an aqueous solution containing appropriate amounts of iron (II) chloride tetrahydrate, iron (III) hexahydrate, and dextran was added dropwise to dried pAm microspheres and the reaction was allowed to incubate for a few hours. The presence of dextran, which is commonly used in two-phase protein extraction processes, in the iron chloride solution is theorized to (1) drive the partitioning of the iron ions into the polymeric scaffold, thereby increasing the iron content inside the hydrogel particles and (2) minimize agglomeration of the resultant iron nanoparticles. The degree of iron loading into the hydrogel was indicated by the hydrogel transitioning from white to orange-yellow (Figure 2(A)). An aqueous solution of ammonium hydroxide was added to the reaction, and the rapid color change from orange-yellow to brown-black signified the formation of the magnetic iron nanoparticles inside and outside of the hydrogel. Retention of the brown-black color of the hydrogels after repeated washes with water is indicative of the strong bond between the iron nanoparticles and the hydrogel. 
Figure 2. Hydrogel particles based on pAm were generated using precipitation polymerization in ethanol using azobisisobutyronitrile (AIBN) as the initiator and N-N'-methylenebis(acrylamide) (BIS) as the cross-linker. Magnetic functionality was introduced to the particles via the in situ coprecipitation of $\mathrm{Fe}(\mathrm{II})$ and $\mathrm{Fe}(\mathrm{III})$ in the polymer network (A); Amine groups are introduced to the magnetic particles via Hofmann degradation (B); Cibacron Blue F3G-A was covalently bound to the aminated magnetic particles to fabricate dye-loaded magnetic hydrogel particles $(\mathbf{C})$.

A

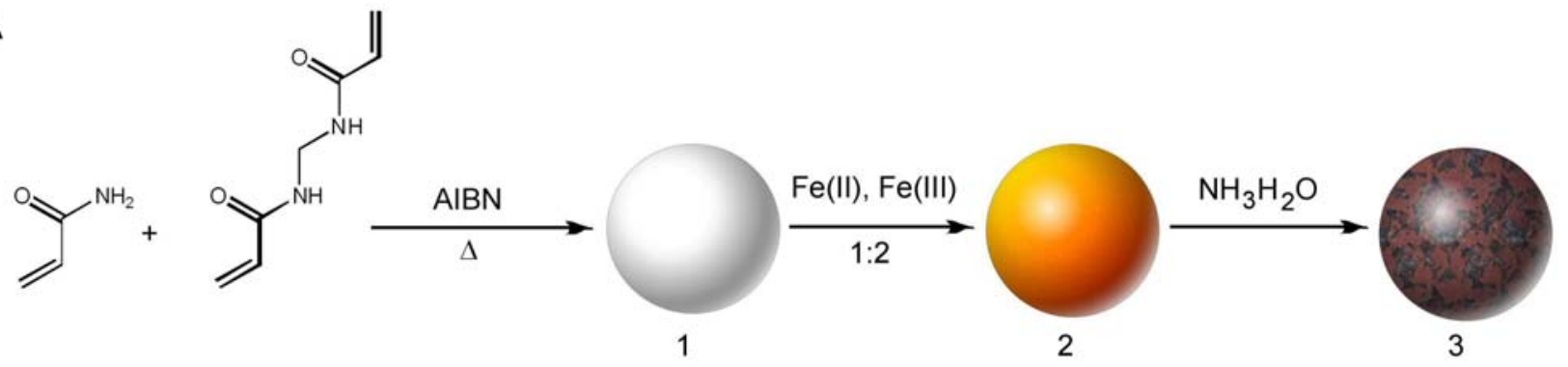

B

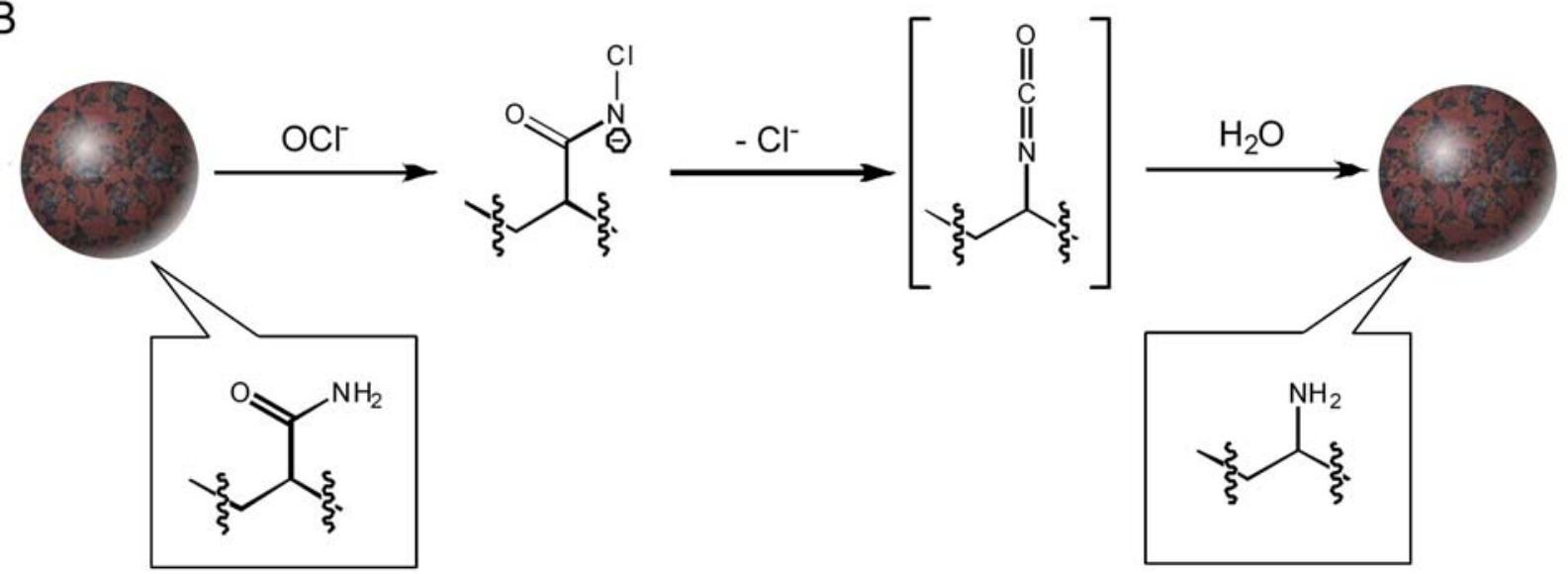

C

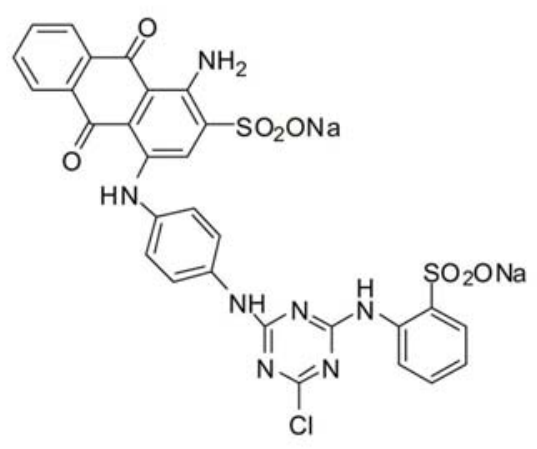

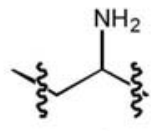

4

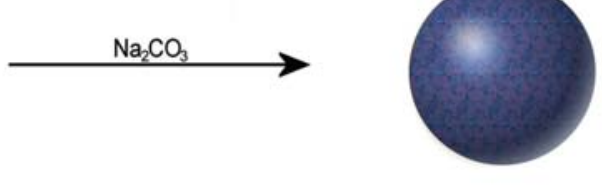

5

Magnetic polymer beads prepared by Ugelstad et al. had an iron content of approximately 10-20\% and were successfully used in cell separation applications [6]. The magnetic particles described in this 
work have an iron content of $78.9 \%$ and are therefore expected to exhibit exceptional performance in separation applications. The total weight of iron oxide nanoparticles per gram of dried magnetic hydrogel $\left(\mathrm{mg} \mathrm{g}^{-1}\right)$ and the percent uptake of iron oxide by the pAm particles are shown in Table 2.

Table 2. Type of hydrogel and total mass of iron oxide per gram of dried magnetic particle.

\begin{tabular}{ccc}
\hline Sample no. & Hydrogel type & Fe weight $\left(\mathbf{m g ~ g}^{\mathbf{- 1}}\right)$ \\
\hline 1 & $50 \mathrm{MHP}$ & 335.9 \\
2 & $75 \mathrm{MHP}$ & 413.8 \\
3 & $100 \mathrm{MHP}$ & 789.9 \\
\hline
\end{tabular}

\subsubsection{Functionalization of Magnetic Hydrogel Particles with Cibacron Blue F3G-A}

Hofmann degradation was employed to introduce amino groups to the polymer backbone of the magnetic pAm hydrogel particles (Figure 2(B)). The reaction was performed at low temperature in order to (1) minimize the tendency of amine-functionalized magnetic hydrogels to aggregate, (2) decrease the reaction rate, and (3) prevent unwanted events such as the hydrolysis of the amido groups and cleavage of the polymeric chains from occurring [13]. Furthermore, oxidation of the resultant amino groups was prevented during the course of the reaction by purging the reaction with nitrogen [13].

The amine-functionalized magnetic pAm particles were further chemically modified with Cibacron Blue F3G-A. The reactive dye was bound to the magnetic hydrogels through a direct reaction between the reactive triazine rings of the dye molecules and the amino groups of the modified pAm magnetic hydrogel particles (Figure 2(C)). The number of moles of dye bound to the 100MHP particles was determined via a comparison of the spectrophotometrically determined number of moles of unbound dye in the combined washes following reaction with the particles and the number of moles of dye present in the original reaction mixture. In order to determine the degree of dye incorporation, a small aliquot $(200 \mu \mathrm{L})$ of the dye-loaded magnetic hydrogel particles was lyophilized and weighed in order to attain the dry weight of the sample. These values were in turn used, along with the total volume of particle suspension, to determine the dye density. By using this method, pAm-based magnetic particles (100MHP) with immobilized Cibacron Blue F3G-A were estimated to have a dye loading of $7.16 \mathrm{mmol}$ of dye per gram $\left(\mathrm{mmol} \mathrm{g}^{-1}\right)$ of dried particle.

\subsection{Particle Swelling Degree and Dynamics}

\subsubsection{Hydration and $\mathrm{pH}$ Studies of Unmodified Magnetic Hydrogel Particles}

Lyophilized aliquots of pAm-based hydrogel and magnetic hydrogel particles were preweighed and suspended in the swelling solvent $\left(\mathrm{dH}_{2} \mathrm{O}\right)$. The samples were left to equilibrate in the swelling solvent at ambient temperature for $24 \mathrm{~h}$. After $24 \mathrm{~h}$, the samples were centrifuged and the excess solvent was removed with a soft tissue before the weight of the swollen hydrogel samples was determined. The influence of different preparations of magnetic hydrogel particles and iron oxide content (50MHP, $75 \mathrm{MHP}$ and 100MHP) on particle swelling capacities is illustrated in Figure 3. An increase in the swelling capacity of the hydrogel was observed with increased iron oxide content in the particle matrix (Figure 3(A)). The fact that particle swelling capacity increases with increased iron oxide loading is 
not surprising. When hydrogels are loaded with iron oxide nanoparticles, the electrostatic forces between the iron oxide nanoparticles and the amide groups of the polymer scaffold contribute to the increased solvation and swelling capacity of the iron-loaded hydrogels relative to plain hydrogels [17]. These interactions and their contribution to the swelling capacity increase as a function of the iron oxide content of the hydrogel particle [17].

The $\mathrm{pH}$ of the swelling solution also has an effect on the swelling capacity of magnetic hydrogels. As illustrated in Figure 3, there is a steady increase in the swelling capacity of the magnetic hydrogel (100MHP) as the $\mathrm{pH}$ rises from 3.0 to 8.0 , while the plain hydrogel shows no $\mathrm{pH}$-responsive behavior.

Figure 3. Comparison of the plain pAm hydrogel particles with the different derivations of the 50MHP (A), 75MHP (B) and 100MHP (C) show an increase in swelling capacity with increasing $\mathrm{pH}$ and increasing iron content. Plain $\mathrm{pAm}$ particles treated with the same conditions used for the in situ iron oxide precipitation (D) also exhibited increased swelling capacity with increasing $\mathrm{pH}$.

A
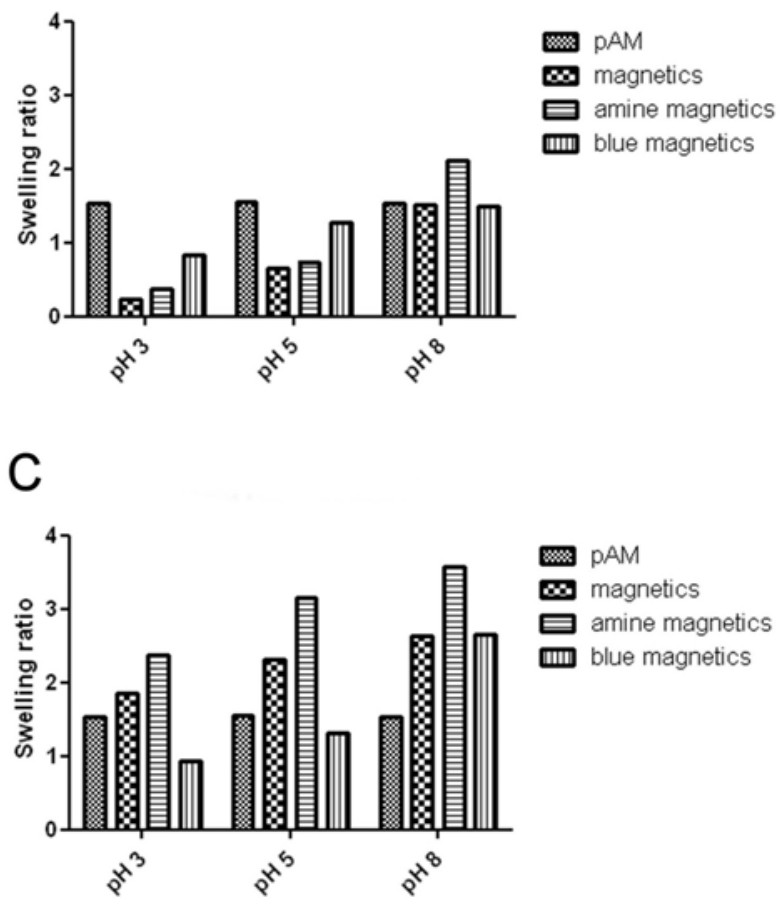

B
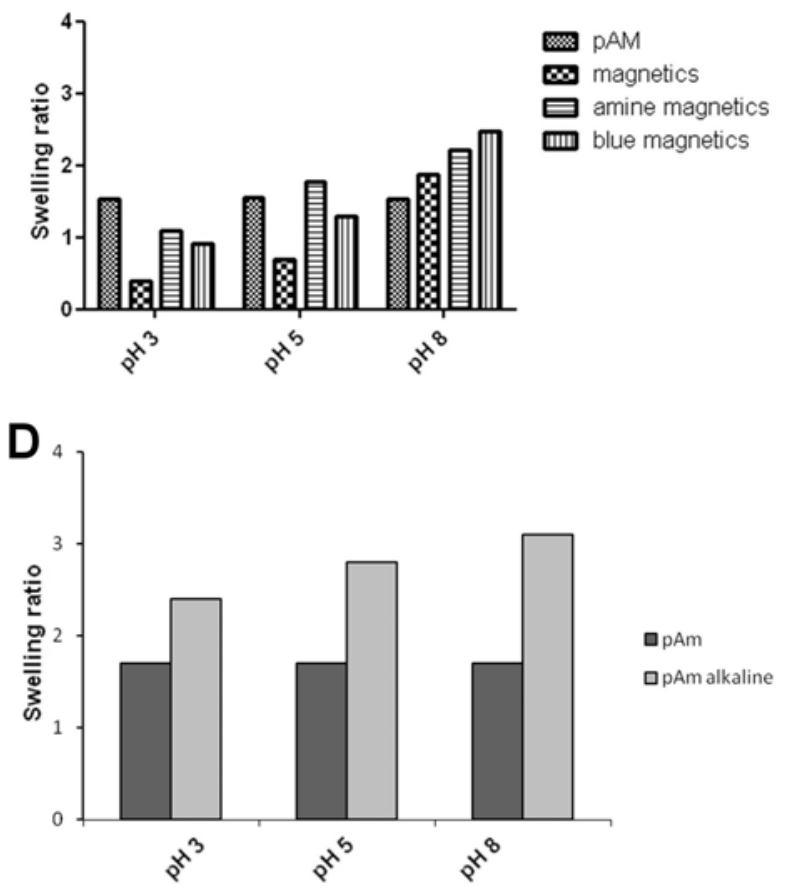

3.2.2. Hydration and $\mathrm{pH}$ Studies of Magnetic Hydrogel Particles

The swelling capacity of three different preparations of magnetic hydrogel particles (underivatized magnetic hydrogel, amine-functionalized magnetic hydrogel, and dye-loaded magnetic hydrogel) was examined (Figure 3). Furthermore, the $\mathrm{pH}$-responsive behavior of the particles was studied in order to determine the impact that the different preparation methods utilized to functionalize the particles had on their hydration behavior. Aliquots of the magnetic hydrogel particles were lyophilized and weighed.

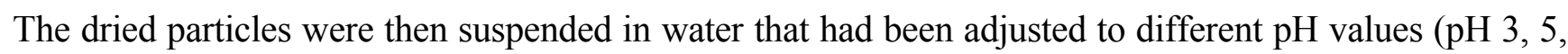
and 8) and allowed to attain equilibrium over $24 \mathrm{~h}$, after which they were centrifuged to separate the particles from the swelling solvent. The excess solvent was removed with a soft tissue before the wet weight of the swollen particles was determined. The results of this study are provided in Figure 3. 


\subsubsection{Underivatized Magnetic Hydrogel Particles}

Figure 3(A) shows the $\mathrm{pH}$-responsive behavior of the underivatized 50MHP, which demonstrated greater swelling as the $\mathrm{pH}$ increased from 3 to 8 . An increase in the overall swelling capacity was noted in the underivatized 75MHP, which contained 23\% more iron oxide than 50MHP (Figure 3(B)). At $\mathrm{pH} 3$, an increase of $62.5 \%$ in the swelling capacity was observed for $75 \mathrm{MHP}$ relative to 50MHP. When the $\mathrm{pH}$ was increased to 5 , the swelling capacity of $75 \mathrm{MHP}$ increased by only $4.5 \%$, although a greater change in the degree of hydration was noted at $\mathrm{pH} 8$, at which the swelling capacity for $75 \mathrm{MHP}$ increased by $23.8 \%$. As shown in Figure 3(C), a greater increase in the swelling capacity was noted for the magnetic particles that were prepared using the $100 \%$ iron salt solution (100MHP), which contained $135 \%$ more iron oxide than 50MHP and 91\% more iron oxide than 75MHP. Relative to $50 \mathrm{MHP}$, an increase of $675 \%$ was noted for the swelling capacity of $100 \mathrm{MHP}$ at $\mathrm{pH} 3$, corresponding to an increase of $377 \%$ relative to $75 \mathrm{MHP}$. At $\mathrm{pH} 5$, the degree of swelling observed for $100 \mathrm{MHP}$ increased by $252 \%$ relative to $50 \mathrm{MHP}$, which corresponds to a $236 \%$ increase with respect to $75 \mathrm{MHP}$. When the $\mathrm{pH}$ is increased to 8 , the swelling capacity of $100 \mathrm{MHP}$ is increased by $74.8 \%$ in relation to 50MHP, which corresponds to an increase of $41.2 \%$ relative to $75 \mathrm{MHP}$.

Significant variation in the swelling capacity of the magnetic hydrogels was noted between magnetic hydrogels containing different amounts of iron oxide. The order of swelling capacity observed in the hydrogel samples at all $\mathrm{pH}$ conditions tested was 50MHP $<75 \mathrm{MHP}<100 \mathrm{MHP}$. This trend can be at least partly explained by considering the aforementioned increased electrostatic forces between the iron oxide nanoparticles and the amide groups of the polymeric backbone. Accordingly, the hydrogel particles containing the greater amount of iron oxide nanoparticles should exhibit the greatest swelling capacity. Furthermore, the alkaline conditions used to precipitate the iron oxide nanoparticles within the hydrogel matrix may introduce carboxyl groups to the hydrogel via partial hydrolysis of the polymer chains [18]. Figure 3(D) shows the $\mathrm{pH}$-responsive behavior of the plain pAm parent hydrogel particles that have been treated with the same conditions used for the in situ precipitation of the iron oxide nanoparticles. The results exhibited in Figure 3(D) may indicate the presence of carboxyl groups in the polymer chain due to the increased swelling capacity as the $\mathrm{pH}$ was increased from 3 to 8 . At low $\mathrm{pH}$ (3), the majority of the carboxyl groups of the partially hydrolyzed pAm particles are in the nonionized state and are more hydrophobic, which results in the collapse of the polymer chain and subsequent decrease in particle size. At high $\mathrm{pH}(8)$, the carboxyl groups are in the ionized state and more hydrophilic, resulting in an increase in the particle size due to (1) the Coulombic interactions between the polymer chains and (2) the osmotic pressure resulting from the counterion influx into the particles $[1,19]$.

\subsubsection{Aminated Magnetic Hydrogel Particles}

Hofmann degradation was used to introduce amine functional groups into the magnetic hydrogel particles. As shown in Figure 3, the amine-functionalized magnetic hydrogel particles exhibited an increasing trend in swelling capacity with increasing $\mathrm{pH}$. It is important to note that potential variability in the degree of amination afforded by the Hofmann degradation reaction complicates direct comparisons between the different aminated magnetic hydrogel particles. Therefore, they were analyzed separately. As shown in Figure 3(A), the aminated 50MHP exhibited a swelling ratio of 0.38 
at $\mathrm{pH}$ 3. When the $\mathrm{pH}$ was increased from 3 to 5 , the degree of hydration was shown to increase by $94.7 \%$. The swelling ratio of the aminated $50 \mathrm{MHP}$ increased by $458 \%$ when the $\mathrm{pH}$ was increased from 3 to 8 , equating to an increase of $187 \%$ in the degree of hydration when the $\mathrm{pH}$ was increased from 5 to 8. At $\mathrm{pH} 3$, the aminated 75MHP exhibited a swelling ratio of 1.10, as shown in Figure 3(B). An increase of $61.8 \%$ was observed for these particles when the $\mathrm{pH}$ was increased from 3 to 5 . When the $\mathrm{pH}$ was increased from 3 to 8 , an increase of $101 \%$ in the swelling ratio was observed for the aminated $75 \mathrm{MHP}$, correlating to an increase of $24.2 \%$ in the degree of hydration when compared to particles at $\mathrm{pH} 5$. At $\mathrm{pH} 3$, the aminated magnetic 100MHP exhibited a swelling ratio of 2.38 , as shown in Figure 3(C). When the $\mathrm{pH}$ was increased from 3 to 5, the degree of hydration for the aminated 100MHP exhibited an increase of $32.4 \%$. An increase of $50.0 \%$ in the swelling ratio was observed for the aminated 100MHP when the $\mathrm{pH}$ was increased from 3 to 8 , corresponding to an increase of $13.3 \%$ in the degree of hydration relative to particles hydrated at $\mathrm{pH} 5$.

The presence of amine functional groups in the hydrogel would be expected to contribute to the collapse of the polymeric backbone at higher $\mathrm{pH}$ values due to the increase in the fraction of neutral unprotonated amine groups, thereby decreasing the swelling ratio. Therefore, over the $\mathrm{pH}$ range tested, the highest degree of hydration is expected at $\mathrm{pH} 3$ and the lowest at $\mathrm{pH}$ 8. However, the opposite trend was observed in the magnetic hydrogel particles, with the highest degree of swelling observed at $\mathrm{pH} 8$ and the lowest at $\mathrm{pH} 3$. This $\mathrm{pH}$-responsive behavior is likely the result of multiple factors, including the presence of the magnetic particles within the particle matrix and the possible presence of carboxyl groups that can be formed as a result of side reactions that can occur during the Hofmann degradation reaction. The initial step in the degradation reaction is the chlorination of the amide group (Figure 2(B)), which should protect the amide group from hydrolysis, a side reaction that results in the formation of the carboxylic acids. The $\mathrm{pH}$-responsive behavior observed in the amine-functionalized magnetic hydrogel particles could reflect the presence of a significant amount of carboxyl groups in the hydrogel, suggesting that not all of the amide groups were chlorinated during the initial step of the Hofmann degradation reaction.

\subsubsection{Dye-Loaded Magnetic Hydrogel Particles}

In order to obtain the Cibacron Blue magnetic hydrogel particles, the reactive affinity dye was bound to the particles via a direct reaction between the reactive triazine rings of the dye molecules and the amino groups in the amine-functionalized magnetic hydrogel particles. The swelling capacity of the dye-loaded magnetic hydrogel particles appears to increase as the $\mathrm{pH}$ of the swelling solution is raised. However, inconsistencies in dye incorporation due to variability in the Hofmann degradation reaction and the degree to which acrylamide amide groups are converted to amines makes it difficult to directly compare the different dye-loaded magnetic hydrogel particles. Therefore, the different dye-loaded magnetic hydrogel particles were analyzed separately.

As shown in Figure 3(A), the dye-loaded 50MHP exhibited a swelling ratio of 0.84 at $\mathrm{pH}$ 3. An increase of $52.4 \%$ was observed in the degree of hydration when the $\mathrm{pH}$ was increased from 3 to 5 . When the $\mathrm{pH}$ was increased from 3 to 8 , an increase of $77.4 \%$ in the swelling ratio was observed for the dye-loaded 50MHP, corresponding to an increase of $16.4 \%$ in the degree of hydration when the $\mathrm{pH}$ was increased from 5 to 8 . At $\mathrm{pH} 3$, the dye-loaded $75 \mathrm{MHP}$ exhibited a swelling ratio of 0.91 , as 
shown in Figure 3(B). When the $\mathrm{pH}$ was increased from 3 to 5, the degree of hydration was shown to increase by $41.8 \%$. When the $\mathrm{pH}$ was increased from 3 to 8 , an increase of $171 \%$ in the swelling ratio was observed for the dye-loaded 75MHP, equivalent to an increase of $91.5 \%$ in the degree of hydration when compared to the particle hydrated at $\mathrm{pH}$ 5. At $\mathrm{pH} 3$, the dye-loaded 100MHP exhibited a swelling ratio of 0.93 , as shown in Figure 3(C). When the $\mathrm{pH}$ was increased from 3 to 5, the dye-loaded 100MHP exhibited an increase of $40.9 \%$ in the degree of hydration. An increase of $186 \%$ in the swelling ratio was observed for the dye-loaded 100MHP when the $\mathrm{pH}$ was increased from 3 to 8 , corresponding to an increase of $103 \%$ in the degree of hydration when the $\mathrm{pH}$ was increased from 5 to 8 .

Due to the multiple acidic sulfonate groups and basic amino groups present on Cibacron Blue F3G-A, the acid-base properties of the reactive affinity dye moieties are complex. Therefore, incorporation of the dye into the magnetic hydrogel particle would be expected to change the acid-base and solvation properties of the particles. Due to their low $\mathrm{pK}_{\mathrm{a}}$, the sulfonate groups will not likely be affected over the $\mathrm{pH}$ range selected for this study, while the protonation state of the secondary aryl amines may vary over the same $\mathrm{pH}$ range. Based on the $\mathrm{pH}$ behavior of core-shell particles with incorporated Cibacron Blue F3G-A [2], it was anticipated that the dye-loaded magnetic hydrogel particles would not exhibit strong $\mathrm{pH}$-responsive behavior. The observed $\mathrm{pH}$ dependence in the hydration of the dye-loaded magnetic hydrogel particles is likely the result of several factors, such as the presence of the magnetite particles within the particle matrix, and the presence of carboxyl groups in the polymer from side-reactions that can arise during the Hofmann degradation reaction.

\subsubsection{Scanning Electron Microscopy (SEM) Analysis}

Scanning electron microscopy (SEM) was used to study the morphology of the prepared pAm-based hydrogel microspheres and the corresponding magnetic hydrogel particles, as shown in Figure 4(A,B), respectively. Both types of particles appear to be monodisperse with an average diameter of approximately $300 \mathrm{~nm}$, suggesting that addition of the iron oxide nanoparticles into the polymeric matrix did not result in an increase in the particle size. Dynamic light scattering revealed a diameter of $289.4 \mathrm{~nm}$ for the pAm particles, which is consistent with the particle sizes shown in the SEM images.

Figure 4. Scanning electron microscopy (SEM) image of pAm-based hydrogel particles (A) prepared via precipitation polymerization in ethanol revealed monodisperse spherical particles. These particles were used to generate magnetic hydrogel particles $(\mathbf{B})$, which were prepared via in situ coprecipitation of iron oxide nanoparticles within the hydrogel matrix.
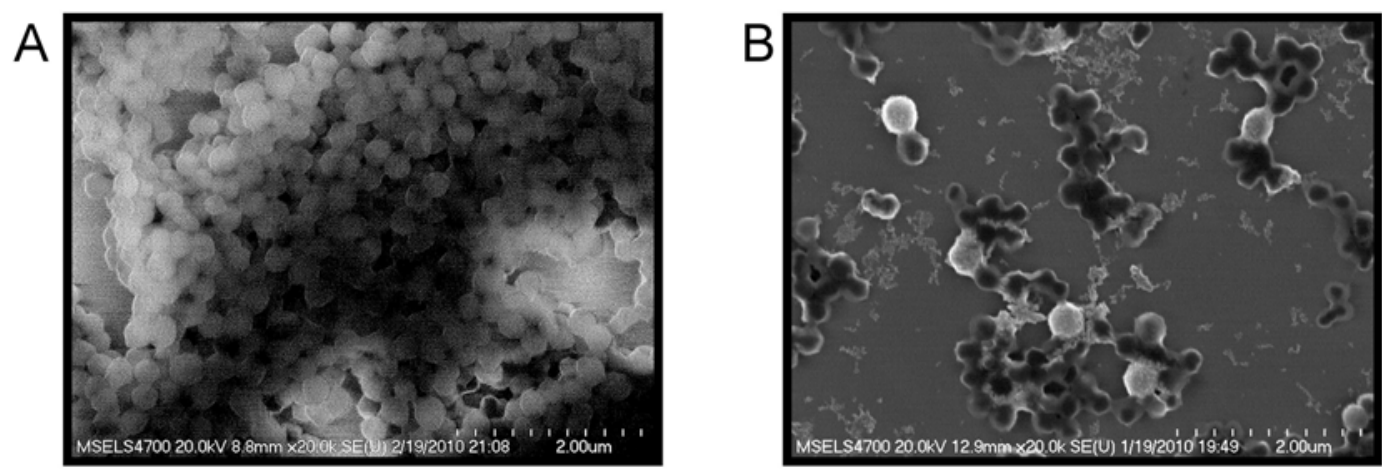


\subsubsection{Sieving Performance}

Core-shell hydrogel particles with incorporated Cibacron Blue F3G-A have demonstrated the ability to bind and concentrate low molecular weight proteins from complex solutions [2]. In order to determine the impact that Cibacron Blue F3G-A incorporation into the polymeric matrix of the magnetic hydrogel particles has on their protein harvesting performance, assorted particles were incubated for $1 \mathrm{~h}$ in solutions containing a defined mixture of reference proteins. SDS-PAGE was used to evaluate the protein uptake capability of the dye-loaded magnetic hydrogel particles. Densitometric analysis of the protein bands in the silver stained gels was used to quantitatively compare the protein harvesting performance of the particles. In this analysis, band intensities were expressed in terms of peak areas using arbitrary units of optical density. The exclusion performance of the magnetic particles was of particular interest, since the presence of high molecular weight, high abundance proteins such as serum albumin can hinder the separation and detection of the lower molecular weight components in biological solutions. In addition to size-based sieving, the particles also need to have the ability to sequester and concentrate proteins with molecular weights of approximately 20,000 Da or less, since this size range corresponds to the molecular weights of physiologically important and informative protein and peptide species present in biological solutions.

Figure 5. SDS-PAGE analysis of dye-loaded core-shell particles and dye-loaded magnetic hydrogel particles incubated with MW markers: (1) MW ladder; (2) model protein solution; (3) supernatant from dye-loaded core-shell particles; (4) bound by dye-loaded core-shell particles; (5) supernatant from dye-loaded magnetic hydrogel particles; (6) bound by dye-loaded magnetic hydrogel particles.

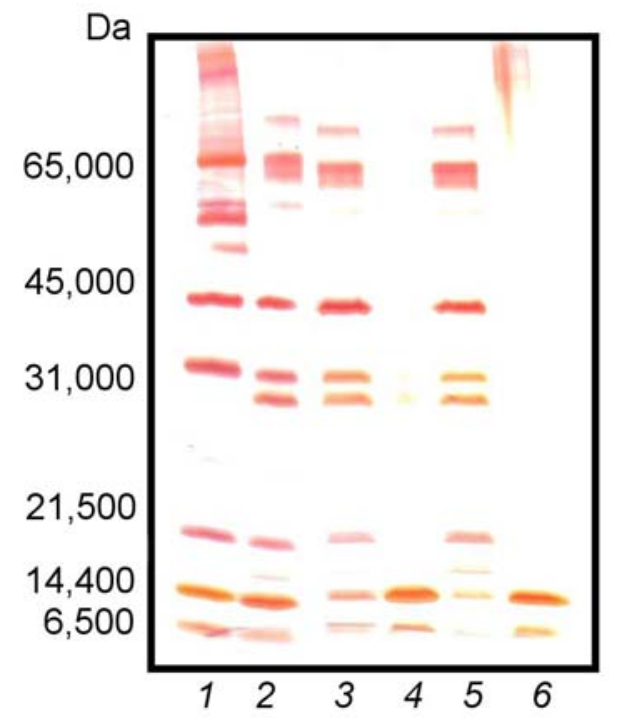

The sieving and harvesting ability of the dye-loaded magnetic hydrogel particles was compared to that of the dye-loaded core-shell particles (Figure 5). After incubation in the protein solution, the dye-loaded core-shell particles exhibited a gel pattern consisting of three bands corresponding to carbonic anhydrase $(31,000 \mathrm{Da})$, lysozyme $(14,400 \mathrm{Da})$ and aprotinin $(6,500 \mathrm{Da})$ with peak areas of $2.95 \times 10^{3}, 4.40 \times 10^{3}$ and $1.35 \times 10^{3}$, respectively (Figure 5, Lane 4). Although the presence of the inert cross-linked pNIPAm shell successfully excluded BSA (66,000 Da) and ovalbumin (45,000 Da), 
the presence of the band corresponding to the 31,000 Da molecular weight region is suggestive of trace uptake or nonspecific binding of carbonic anhydrase. The gel pattern for the dye-loaded magnetic hydrogel particles exhibited two prominent bands on the silver stained gel, with densitometric analysis indicating a $2.70 \%$ increase in lysozyme binding and a $15.4 \%$ increase in aprotinin binding relative to the core-shell particles (Figure 5, Lane 6). The exceptional sieving properties of the dye-loaded magnetic particle in excluding larger protein species likely reflect the contribution of the higher degree of cross-linking present in the acrylamide hydrogel framework.

The harvesting performances of the different intermediate particles and that of the magnetic particles were compared by incubating the particles in solutions containing a defined mixture of proteins for $1 \mathrm{~h}$ (Figure 6). The gel pattern exhibited by plain pAm particles consisted of only one band corresponding to lysozyme with a peak area of $1.19 \times 10^{3}$ (Figure 6, Lane 4). When the pAm particles were loaded with iron oxide nanoparticles, the amount of lysozyme harvested by the particles increased by $86.5 \%$ (Figure 6, Lane 6). In addition to lysozyme, the magnetic hydrogel particles also harvested aprotinin (peak area $1.66 \times 10^{3}$ ) while exhibiting near complete exclusion of BSA (peak area 57.657). When the magnetic hydrogel particles were functionalized with amine groups, lysozyme harvesting increased by $146 \%$ with respect to the plain pAm particles, corresponding to a $31.7 \%$ increase relative to the magnetic hydrogel particles (Figure 6, Lane 8). Furthermore, the amine-functionalized magnetic hydrogel particles harvested $124 \%$ more aprotinin and exhibited $49.8 \%$ less nonspecific BSA binding relative to the unmodified magnetic hydrogel particles. By incorporating Cibacron Blue F3G-A into the amine-functionalized magnetic hydrogel particles, lysozyme binding increased by $501 \%$ relative to the plain pAm particles (Figure 6, Lane 10). In comparison to the magnetic hydrogel particles, magnetic hydrogel particles with incorporated Cibacron Blue F3G-A harvested $222 \%$ more lysozyme and $275.5 \%$ more aprotinin, and relative to the amine-functionalized magnetic hydrogel particles, the dye-loaded magnetic hydrogel particles harvested 145\% more lysozyme and $67.8 \%$ more aprotinin. In addition to the dramatic increase in lysozyme and aprotinin harvesting, the dye-loaded magnetic hydrogel particles also exhibited near complete exclusion of BSA and other high molecular weight species present in the test solution. These results suggest that incorporation of the reactive affinity dye into the particle structure has a substantial positive impact on the harvesting performance of the particles for proteins with MW of 14,400 Da or less.

Even though silver staining affords sensitivity that is superior to that of traditional staining methods using Coomassie Brilliant Blue, making it well suited for determining the sequestration/exclusion capabilities of the particles, it cannot be used to quantitatively compare different proteins on the same gel due to potential inconsistencies in the resultant band intensities. That being said, the results of these studies indicate that inclusion of dye within the magnetic particle matrix translates in improved harvesting efficiency for low molecular weight proteins and peptides. Furthermore, the results suggest that incorporation of the affinity dye provides the particles with the ability to more effectively exclude higher molecular weight proteins and peptides, although whether this phenomenon is the result of electrostatics, crowding or both has yet to be determined. 
Figure 6. SDS-PAGE analysis of dye-loaded magnetic and intermediate pAm particles with varied iron oxide content incubated with MW markers: (1) MW ladder; (2) model protein solution; (3) supernatant from pAm particles; (4) bound by pAm particles; (5) supernatant from 100MHP; (6) bound by 100MHP; (7) supernatant from aminated 100MHP; (8) bound by aminated 100MHP; (9) supernatant from dye-loaded 100MHP; (10) bound by dye-loaded 100MHP.

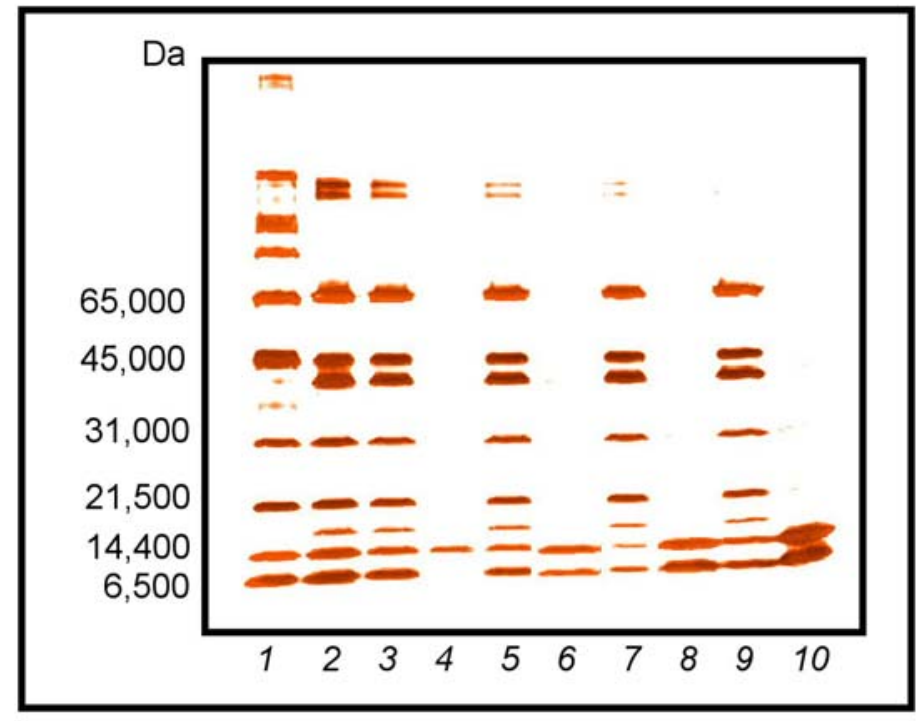

\section{Conclusions}

In the present study, Cibacron Blue F3G-A was incorporated into pAm-based hydrogel particles containing iron oxide nanoparticles in order to fabricate dye-loaded magnetic hydrogel particles. A simple technique was used to synthesize pAm-based microspheres with uniform size distributions via precipitation polymerization in ethanol without the use of dispersing agents. While the magnetic, aminated magnetic, and dye-loaded magnetic microspheres exhibited the ability to sequester and concentrate low molecular weight proteins from the test solutions; the presence of Cibacron Blue F3G-A significantly enhanced the protein harvesting capacity of the particles. Overall, the magnetic pAm particles containing Cibacron Blue F3G-A demonstrated efficient harvesting and size-based sieving under the conditions tested. Furthermore, the sulfonate groups present on the dye enhanced the solvation properties of the dye-incorporated magnetic particles, thereby improving the overall stability of the magnetic particles. The results presented here suggest the potential that these dye-incorporated particles have as tools for biomarker harvesting and other biomedical separation applications.

\section{Acknowledgements}

The authors appreciate the generous support of Vikas Chandhoke and the College of Science at George Mason University. This research was supported in part by the Italian Istituto Superiore di Sanità in the framework of the Italy/USA cooperation agreement between the U.S. Department of Health and Human Services, George Mason University, and the Italian Ministry of Public Health. In addition, this research was also partially supported by the U.S. Department of Energy (grant number DE-FC52-04NA2545) and the NIH/NCI (grant number 1R21CA137706-02). 


\section{References}

1. Luchini, A.; Geho, D.H.; Bishop, B.; Tran, D.; Xia, C.; Dufour, R.L.; Jones, C.D.; Espina, V.; Patanarut, A.; Zhou, W.; et al. Smart hydrogel particles: Biomarker harvesting: One-step affinity purification, size exclusion, and protection against degradation. Nano Lett. 2008, 8, 350-361.

2. Patanarut, A.; Luchini, A.; Botterell, P.J.; Mohan, A.; Longo, C.; Vorster, P.; Petricoin, E.F.; Liotta, L.A.; Bishop, B. Synthesis and characterization of hydrogel particles containing Cibacron Blue F3G-A. Colloids Surf. A 2010, 362, 8-19.

3. Mackova, H.; Kralova, D.; Horak, D. Magnetic poly(N-isopropylacrylamide) microspheres by dispersion and inversion emulsion polymerization. J. Polym. Sci. A: Polym. Chem. 2007, 45, 5884-5898.

4. Zhang, F.; Wang, C.-C. Preparation of p(NIPAm-co-AA) microcontainers surface-anchored with magnetic nanoparticles. Langmuir 2009, 25, 8255-8262.

5. Liu, H.; Wang, C.; Gao, Q.; Liu, X.; Tong, Z. Magnetic hydrogels with supracolloidal structures prepared by suspension polymerization stabilized by $\mathrm{Fe}_{2} \mathrm{O}_{3}$ nanoparticles. Acta Biomater. 2010, 6, 275-281.

6. Ugelstad, J.; Berge, A.; Ellingsen, T.; Schmid, R.; Nilsen, T.-N.; Mork, P.C.; Stenstad, P.; Hornes, E.; Olsvik, O. Preparation and application of new monosized polymer particles. Prog. Polym. Sci. 1992, 17, 87-161.

7. Bajpai, A.K.; Bajpai, S.K. Kinetics of polyacrylamide adsorption at the iron oxide-solution interface. Colloids Surf. A 1995, 101, 21-28.

8. Sivudu, K.S.; Rhee, K.Y. Preparation and characterization of $\mathrm{pH}$-responsive hydrogel magnetite nanocomposite. Colloids Surf. A 2009, 349, 29-34.

9. Lee, L.T.; Somasundaran, P. Adsorption of polyacrylamide on oxide minerals. Langmuir 1989, 5, 854-860.

10. McGuire, M.J.; Addai-Mensah, J.; Bremmell, K.E. Spectroscopic investigation of the adsorption mechanisms of polyacrylamide polymers onto iron oxide particles. J. Colloid Interface Sci. 2006, 299, 547-555.

11. Kawaguchi, H.; Fujimoto, K.; Nakazawa, Y.; Sakagawa, M.; Ariyoshi, Y.; Shidara, M.; Okazaki, H.; Ebisawa, Y. Modification and functionalization of hydrogel microspheres. Colloids Surf. A 1996, 109, 147-154.

12. Aoyagi, J.; Suzuki, T.; Kato, R.; Nagata, M. Microsphere and Method for Production Thereof. U.S. Patent 5,635,574, 3 June 1997.

13. Hong, J.; Xu, D.; Gong, P.; Sun, H.; Dong, L.; Yao, S. Covalent binding of a-chymotrypsin on the magnetic nanogels covered by amino groups. J. Mol. Catal. B: Enzym. 2007, 45, 84-90.

14. Fredolini, C.; Meani, F.; Reeder, K.A.; Rucker, S.; Patanarut, A.; Botterell, P.J.; Bishop, B.; Longo, C.; Espina, V.; Petricoin, E.F.; et al. Concentration and preservation of very low abundance biomarkers in urine, such as human growth hormone (hGH), by Cibacron Blue F3G-A loaded hydrogel particles. Nano Res. 2008, 1, 502-518.

15. Brahim, S.; Narinesingh, D.; Guiseppi-Elie, A. Synthesis and hydration properties of $\mathrm{pH}$-sensitive p(HEMA)-based hydrogels containing 3-(trimethoxysilyl)propyl methacrylate. Biomacromolecules 2003, 4, 497-503. 
16. Budavari, S. The Merck Index: An Encyclopedia of Chemicals, Drugs, and Biological, 12th ed.; Merck: Whitehouse Station, NJ, USA, 1996.

17. Ni, H.; Kawaguchi, H. Mechanism of preparing monodisperse poly(acrylamide/methacrylic acid) microspheres in ethanol. I. J. Polym. Sci. A: Polym. Chem. 2004, 42, 2833-2844.

18. Zeynali, M.E.; Rabbii, A. Alkaline hydrolysis of polyacrylamide and study on poly(acrylamideco-sodium acrylate) properties. Iran. Polym. J. 2002, 11, 269-275.

19. Xia, X.; Hu, Z. Synthesis and light scattering study of microgels with interpenetrating polymer networks. Langmuir 2004, 20, 2094-2098.

(C) 2011 by the authors; licensee MDPI, Basel, Switzerland. This article is an open access article distributed under the terms and conditions of the Creative Commons Attribution license (http://creativecommons.org/licenses/by/3.0/). 